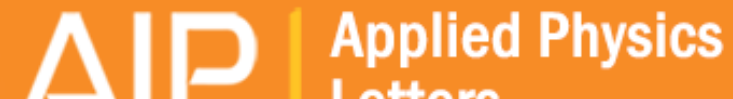 Letters
}

\section{Force-controlled spatial manipulation of viable mammalian cells and micro- organisms by means of FluidFM technology}

Pablo Dörig, Philipp Stiefel, Pascal Behr, Edin Sarajlic, Daniel Bijl, Michael Gabi, János Vörös, Julia A.

Vorholt, and Tomaso Zambelli

Citation: Applied Physics Letters 97, 023701 (2010); doi: 10.1063/1.3462979

View online: http://dx.doi.org/10.1063/1.3462979

View Table of Contents: http://scitation.aip.org/content/aip/journal/apl/97/2?ver=pdfcov

Published by the AIP Publishing

\section{Articles you may be interested in}

A Mach-Zender digital holographic microscope with sub-micrometer resolution for imaging and tracking of marine micro-organisms

Rev. Sci. Instrum. 85, 123113 (2014); 10.1063/1.4904449

Mixing enhancement by biologically inspired convection in a micro-chamber using alternating current galvanotactic control of the Tetrahymena pyriformis

Appl. Phys. Lett. 103, 103703 (2013); 10.1063/1.4820584

Controlled manipulation and actuation of micro-objects with magnetotactic bacteria

Appl. Phys. Lett. 89, 233904 (2006); 10.1063/1.2402221

In situ imaging of micro-organisms in intense magnetic fields

Rev. Sci. Instrum. 76, 103706 (2005); 10.1063/1.2103427

Two-dimensional tracking of a motile micro-organism allowing high-resolution observation with various imaging techniques

Rev. Sci. Instrum. 76, 034301 (2005); 10.1063/1.1857632

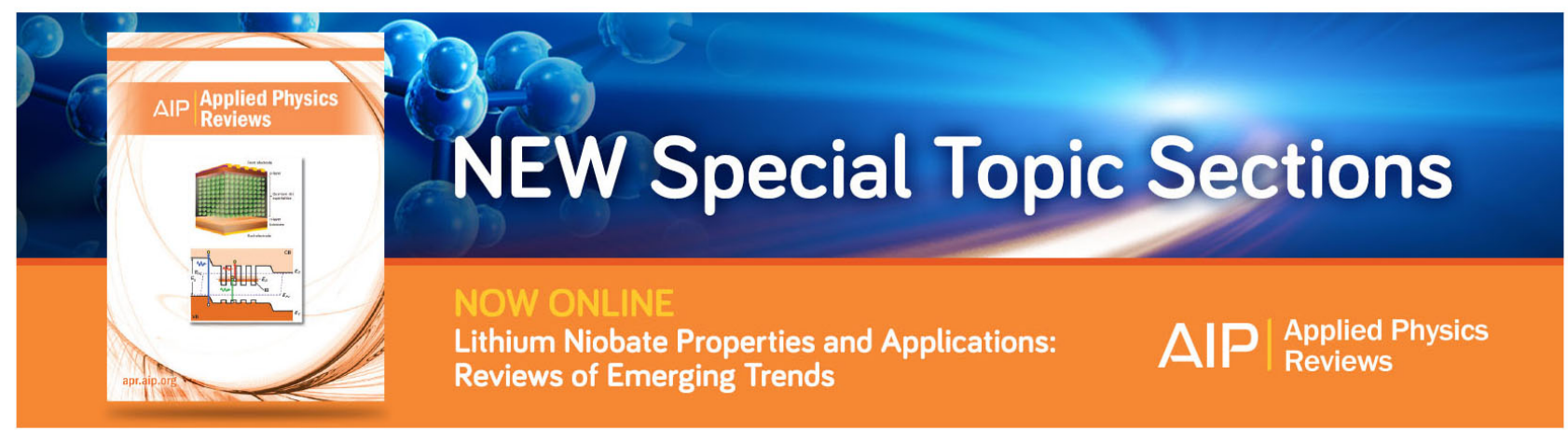




\title{
Force-controlled spatial manipulation of viable mammalian cells and micro-organisms by means of FluidFM technology
}

\author{
Pablo Dörig, ${ }^{1}$ Philipp Stiefel, ${ }^{2}$ Pascal Behr, ${ }^{1,3}$ Edin Sarajlic, ${ }^{3,4}$ Daniel Bijl, ${ }^{3,4}$ Michael Gabi, ${ }^{1,3}$ \\ János Vörös, ${ }^{1}$ Julia A. Vorholt, ${ }^{2}$ and Tomaso Zambelli ${ }^{1, a)}$ \\ ${ }^{1}$ Laboratory of Biosensors and Bioelectronics, Institute for Biomedical Engineering, ETH Zurich, \\ 8092 Zurich, Switzerland \\ ${ }^{2}$ Institute of Microbiology, ETH Zurich, 8093 Zurich, Switzerland \\ ${ }^{3}$ Cytosurge LLC, Löwenstrasse 42, 8001 Zurich, Switzerland \\ ${ }^{4}$ SmartTip BV, 7500 AH Enschede, The Netherlands
}

(Received 3 March 2010; accepted 15 April 2010; published online 12 July 2010)

\begin{abstract}
The FluidFM technology uses microchanneled atomic force microscope cantilevers that are fixed to a drilled atomic force microscope cantilevers probeholder. A continuous fluidic circuit is thereby achieved extending from an external liquid reservoir, through the probeholder and the hollow cantilever to the tip aperture. In this way, both overpressure and an underpressure can be applied to the liquid reservoir and hence to the built-in fluidic circuit. We describe in this letter how standard atomic force microscopy in combination with regulated pressure differences inside the microchanneled cantilevers can be used to displace living organisms with micrometric precision in a nondestructive way. The protocol is applicable to both eukaryotic and prokaryotic cells (e.g., mammalian cells, yeasts, and bacteria) in physiological buffer. By means of this procedure, cells can also be transferred from one glass slide to another one or onto an agar medium. (C) 2010 American Institute of Physics. [doi:10.1063/1.3462979]
\end{abstract}

The ability to obtain information at the single-cell level is becoming of central importance for numerous biological questions and represents a major challenge. ${ }^{1}$ Innovative technologies are required to address individual cells, whereby to "address" has to be conceived in broad manner ranging from displacement to injection up to downstream analysis.

In the present work we will focus on the controlled spatial displacement of single cells. Already 20 years ago, Ashkin $e t$ al. pioneered the development of the optical tweezers as a mean to manipulate biological objects. ${ }^{2-7}$ Exploiting the trapping forces due to the radiation pressure through intense and collimated lasers ${ }^{8,9}$ viruses, bacteria as well as cellular organelles could be displaced in a controlled way. However, optical tweezers might damage cells, ${ }^{10-12}$ and are not appropriate for detaching adherent, spread cells from surfaces. The latter also holds for glass micropipettes, ${ }^{13-15}$ the oldest instrument to manipulate single organisms.

Atomic force microscope (AFM) (Ref. 16) can provide the required force feedback in the piconewton range for gentle and accurate manipulation compared to the micronewton range ${ }^{17}$ of glass micropipettes. With respect to the manipulation of organisms, it is already widely employed for a bright spectrum of adhesion experiments. ${ }^{18-23}$ So far AFM has not been used for displacement experiments because organisms are readily attached to the underside of the AFM cantilever preventing their release onto another position of the substrate. The development of the FluidFM technology ${ }^{24}$ combining the precise AFM force feedback with nanofluidics via an incorporated microchannel directly in the cantilever opens novel strategies for the spatial manipulation of biological objects. The microchannel inside the cantilever ends with a submicron aperture at the apex of the pyramidal tip while the other end leads to a reservoir. By fixing the chip against the hollow probeholder, a continuous fluidic path is

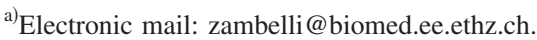

obtained connecting the tip aperture with the reservoir which in turn can be connected to a pressure controller. Due to the closed fluidic path, the technique can also be used in a completely liquid environment as required in biology.

In this study, we demonstrate an unprecedented way of spatially manipulating individual cells. We take advantage of the unique features of the FluidFM technology: On the one hand the AFM force feedback control for a safe and quick approach onto the biological object of interest, and on the other hand the possibility to apply controlled pressure differences to "grasp" the cells [Figs. 1(a) and 1(b)], whereby different types of tip apertures can be used. The object is lifted up and moved to a chosen predefined position where the force feedback control is activated again to reengage the surface and a short overpressure pulse is administered to release the object [Figs. 1(c) and 1(d)].

We used a custom built AFM platform inspired by a work of the Gaub's group. ${ }^{25}$ All the noncustom components (laser, photodetector, z-piezo, and stepper-motor) were pur-
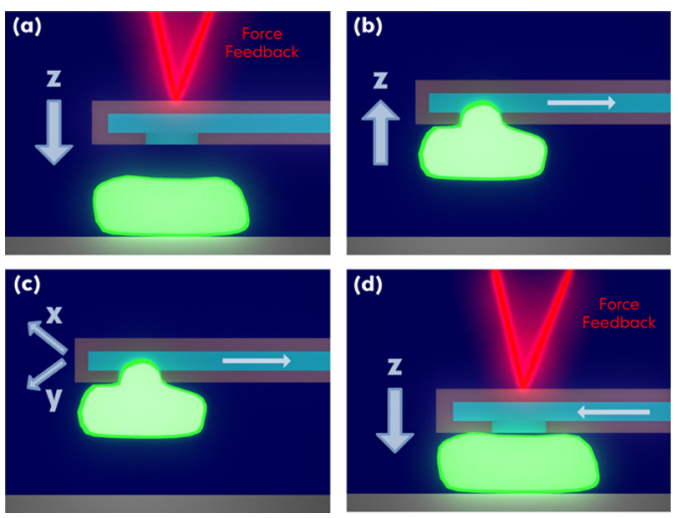

FIG. 1. (Color online) Scheme of the manipulation procedure in liquid environment. (a) Force-controlled approach onto the cell; (b) application of underpressure and lifting up; (c) x-y displacement; (d) force-controlled landing at the new position and release with an overpressure pulse. 


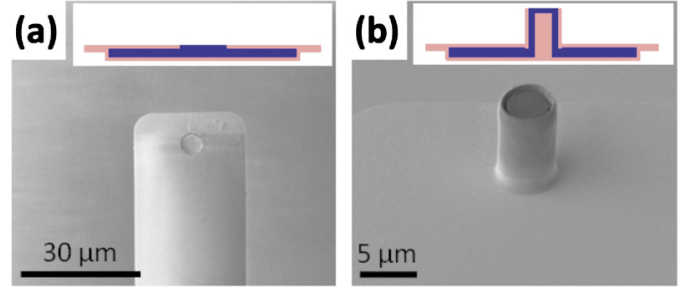

FIG. 2. (Color online) Microchanneled AFM cantilevers. Scanning electron microscopy images (a) of a blunt cantilever (150 $\mu \mathrm{m}$ long, $\mathrm{k} \sim 3 \mathrm{~N} \mathrm{~m}^{-1}$ ) and (b) of a double-tube tip cantilever. Both with a cartoon of the transversal section at the aperture (light: $300 \mathrm{~nm}$ thick silicon nitride walls, dark: liquid filling the $25 \mu \mathrm{m}$ wide and $1 \mu \mathrm{m}$ shallow channel).

chased from Thorlabs (Germany) and operated by a single controller unit via LabView based software. ${ }^{26}$ The AFM thereby adjusts the position only in the $\mathrm{z}$ direction whereas movement on the $\mathrm{x}-\mathrm{y}$ plane is achieved with micrometric screws mounted on the optical microscope stage. A new generation of hollow AFM cantilevers ${ }^{27-31}$ were especially designed for displacement experiments having the aperture directly on the underside of the cantilever close to its end (blunt cantilevers, Fig. 2(a), Cytosurge, Switzerland). The hollow cantilever with an integrated microchannel and a fluidic reservoir, was microfabricated in a wafer-scale process similar to the one proposed by Deladi et al. ${ }^{27,32}$ The fabrication process is based on silicon surface micromachining and bulk glass micromachining by powder blasting. The process starts on a silicon substrate coated by a silicon nitride layer. In this layer a microscale opening that will later serve as an aperture at the tip of the microchanneled cantilever is defined by reactive ion etching (RIE). Next, a sacrificial polysilicon layer is deposited and patterned to form the layout of the microchannel. The patterned polysilicon layer was then encapsulated by deposition of another silicon nitride layer. After the encapsulation, the cantilever beams and a relatively large inlet on the opposite end of the prospective microchannel were formed by RIE of the top silicon nitride layer. In the next fabrication step, a glass wafer, in which holes connecting both wafer sides (through holes) are powder-blasted, was aligned and anodically bonded to the silicon substrate. The glass wafer thereby also serves as the mechanical support for the cantilever and the microchannels. It holds the through holes too that serve as the inlet reservoirs. After the bonding, the wafer-stack was immersed into a silicon etchant (TMAH) to completely release the cantilevers and at the same time to empty the microchannels by removing the sacrificial polysilicon layer through the inlet holes and the cantilever aperture. The basic fabrication sequence can be slightly modified in order to substitute the aperture at the end of the cantilever with a double tube-like tip, as the one in Fig. 2(b). ${ }^{26}$

To prove the validity of our strategy and the versatility of our system we carried out displacement experiments with three different kinds of viable cells: mammalian cells (neurons with a typical size of $\sim 20 \mu \mathrm{m}$ ), yeasts (Saccharomyces cerevisiae, with a typical diameter of $\sim 8 \mu \mathrm{m}$ ), and bacteria (Escherichia coli with a typical length of $\sim 3 \mu \mathrm{m}$ and a diameter of $\sim 1.5 \mu \mathrm{m}$ ). Figure 3(a) is a brightfield image of S. cerevisiae adsorbed on a glass slide in $0.9 \% \mathrm{NaCl}$ after the manipulation procedure. As typical for budding yeasts, $S$. cerevisiae are often found as couples of mother and daughter (bud) cells. The hollow cantilever was filled with $0.9 \% \mathrm{NaCl}$, optically positioned over a budding yeast ${ }_{S}$ cell and ap-

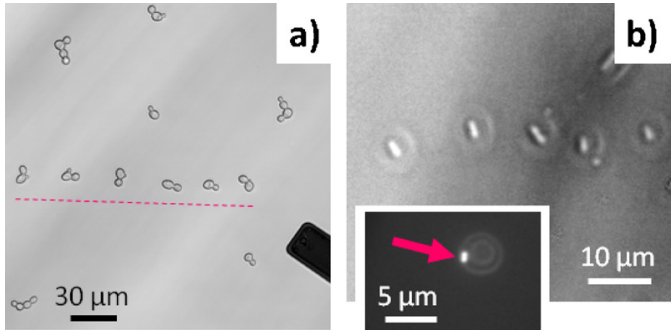

FIG. 3. (Color online) Spatial manipulation of single micro-organisms. (a) Brightfield image of budding $S$. cerevisiae cells after manipulation to form a line of six cells (Ref. 26 for the corresponding image before the manipulation); (b) Brightfield image of a line of five displaced $E$. coli cells. The inset is a fluorescent image of a green fluorescent protein expressing bacterial cell (Methylobacterium extorquens, arrow) aspirated by a double-tube tip.

proached in contact mode taking advantage of the AFM force feedback. Once the cantilever was in contact with the cell, an underpressure of $\sim 50$ mbar was applied in the fluidic channel by means of a $10 \mathrm{ml}$ syringe. As a consequence, the cell was sucked against the channel aperture. It was then lifted by retracting the cantilever with a stepper motor and displaced in the x-y plane with the micrometric screws of the microscope stage over the optically chosen new position. It was deposited onto the substrate with an AFM approach in contact mode and finally released by applying a short overpressure pulse with the syringe while retracting the piezo. Serial manipulations were possible using a single cantilever.

For the displacement of bacteria another tip design was used consisting of two concentrically cylinders separated by $\sim 1 \mu \mathrm{m}$ [Fig. 2(b)]. The gap was small enough so that bacteria remain on the base of the internal cylinder instead of being aspired into the microchannel [inset of Fig. 3(b)]. Bacterial motility on the substrate was hindered by a poly(L-lysine) coating of the glass slide, whereas the cantilever was functionalized with a layer of poly(L-lysine)- $g$-poly(ethylene glycol) (PLL-g-PEG), a polymer known for its simplicity and effectiveness in resisting the absorption of biomolecules. ${ }^{33}$ Figure $3(\mathrm{~b})$ shows five $E$. coli cells spatially displaced one after the other in order to be arranged along a virtual line.

The same manipulation scheme could be applied to adherent, spread mammalian cells as demonstrated in Fig. 4. The external and internal surfaces of the channeled cantilevers were functionalized with PLL-g-PEG, the glass slide with fibronectin while the channel was filled with phosphatebuffered saline. Figure 4(a) shows two interconnected neuronal cells by a tubular structure (neurite, light arrow). After being sucked against the $6 \mu \mathrm{m}$ aperture of a blunt cantilever (dashed circle), one neuron was detached from the glass slide [Fig. 4(b)], displaced on the x-y plane [Fig. 4(c)], and redeposited onto the substrate [Fig. 4(d)]. Curiously, the neurite remained intact during the manipulation so that the transmitted traction resulted in a partially detachment the other neuron and loosening of one anchor point (dark arrow). Neurons were kept attached to the aperture by applying a continuous underpressure of $\sim 300$ mbar. From corresponding deflection vs. distance curves ${ }^{34}$ it was inferred that a force of roughly $1.0 \pm 0.3 \mu \mathrm{N}$ had to be overcome to detach neurons in such conditions which is in the range of nano to microNewton reported in the literature. . $^{35,36}$

With this procedure, cell displacement is also feasible from one glass slide to another one or onto an agar medium, achievement precluded to optical tweezers. Organisms re. 


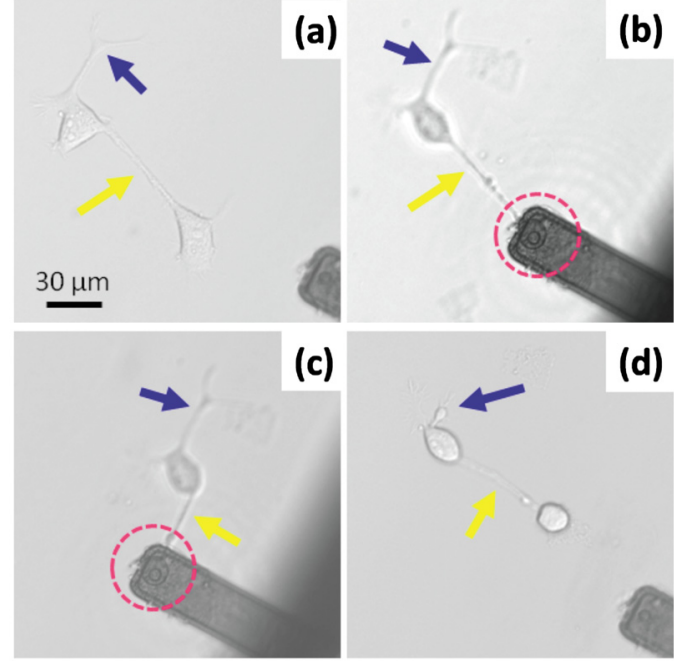

FIG. 4. (Color online) Sequential brightfield images of the spatial manipulation of a NG108 neuroblastoma cell on a glass slide coated with fibronectin. (a) Two neuronal cells interconnected by a neurite (light arrow, while the dark arrow indicates an anchoring projection); (b) detachment of one cell which is aspirated at the cantilever aperture (dashed circle); (c) x-y cell displacement; (d) cell redeposition on the glass slide. In (b) and (c) the optical focus is set on the detached cell. Force-controlled approach and aspiration took only few seconds each, while the delicate $30 \mu \mathrm{m}$ lift-up took $5 \mathrm{~min}$ as the stepper motor was operated with steps of $100 \mathrm{~nm}$ per second.

main attached at the aperture of the cantilever in air during the few tens of seconds necessary to remove the original slide and position a new one under the AFM. As a proof of principle, we grasped yeast cells in liquid and placed them onto glass cover slides coated with a $1 \mu \mathrm{m}$ layer of agar. After proving the force-assisted spatial displacement of viable cells, we verified that the manipulation does not damage them by monitoring their reproduction activity.

In conclusion, we have shown that the FluidFM technology is suitable to carry out spatial manipulation of individual viable eukaryotic and prokaryotic cells. Cells are aspirated to the aperture at the end of the microchanneled cantilever by applying an underpressure so that they can be released at the new position simply upon reverse application of an overpressure pulse. The displacement procedure is supported by the AFM force-feedback ensuring that it can be carried out in a serial fashion and that the organisms are not damaged during the manipulation. Applications are to be contemplated in several different directions. Individual cells can be placed in particular regions (e.g., microwells) of a substrate for singlecell PCR amplification or neurons onto nodal points of electrical circuits for investigations of neuronal networks. Cells can also be selected from environmental samples and deposited individually for clonal reproduction. Furthermore, cells can be attached to the end of a cantilever in a simple and straightforward way for cell-adhesion AFM experiments circumventing the need for often extremely time-consuming biochemical functionalization.

P.D. and P.S. equally contributed to this work. We are indebted to Martin Erdmann (LMU Munich, Germany) and Jérôme Polesel Maris (CEA Saclay, France) for fruitful discussions concerning the design of a custom AFM, to Dominik Textor and the students of the PPS and Gruppenarbeit "Let's build our own bioAFM!" (Filipe Barata, David Handschin, Michael Kleint, Konstantin Kunz, Lisa Meier, Stefan Schneider, and Thomas Walti. ETH D-ITET) for the development of the custom-built AFM platform, and finally to Stephen Wheeler and Martin Lanz (ETH LBB workshop) for technical support.

${ }^{1}$ D. Di Carlo and L. P. Lee, Anal. Chem. 78, 7918 (2006).

${ }^{2}$ A. Ashkin and J. M. Dziedzic, Science 235, 1517 (1987).

${ }^{3}$ A. Ashkin, J. M. Dziedzic, and T. Yamane, Nature (London) 330, 769 (1987).

${ }^{4}$ A. Ashkin and J. M. Dziedzic, Proc. Natl. Acad. Sci. U.S.A. 86, 7914 (1989).

${ }^{5}$ G. Weber and K. O. Greulich, Int. Rev. Cytol. 133, 1 (1992).

${ }^{6}$ K. Svoboda and S. M. Block, Annu. Rev. Biophys. Biomol. Struct. 23, 247 (1994).

${ }^{7}$ P. Jordan, J. Leach, M. Padgett, P. Blackburn, N. Isaacs, M. Goksor, D Hanstorp, A. Wright, J. Girkin, and J. Cooper, Lab Chip 5, 1224 (2005).

${ }^{8}$ A. L. Weisenhorn, P. K. Hansma, T. R. Albrecht, and C. F. Quate, Appl. Phys. Lett. 54, 2651 (1989).

${ }^{9}$ W. H. Wright, G. J. Sonek, and M. W. Berns, Appl. Phys. Lett. 63, 715 (1993).

${ }^{10}$ K. König, H. Liang, M. W. Berns, and B. J. Tromberg, Opt. Lett. 21, 1090 (1996).

${ }^{11}$ K. C. Neuman, E. H. Chadd, G. F. Liou, K. Bergman, and S. M. Block, Biophys. J. 77, 2856 (1999).

${ }^{12}$ M. B. Rasmussen, L. B. Oddershede, and H. Siegumfeldtl, Appl. Environ. Microbiol. 74, 2441 (2008).

${ }^{13}$ T. K. Chowdhury, J. Phys. E 2, 1087 (1969).

${ }^{14}$ R. M. Hochmuth, J. Biomech. 33, 15 (2000).

${ }^{15}$ D. Sanchez, N. Johnson, C. Li, P. Novak, J. Rheinlaender, Y. J. Zhang, U. Anand, P. Anand, J. Gorelik, G. I. Frolenkov, C. Benham, M. Lab, V. P. Ostanin, T. E. Schaffer, D. Klenerman, and Y. E. Korchev, Biophys. J. 95, 3017 (2008).

${ }^{16}$ G. Binnig, C. F. Quate, and C. Gerber, Phys. Rev. Lett. 56, 930 (1986).

${ }^{17}$ P. H. Tsang, G. L. Li, Y. V. Brun, L. Ben Freund, and J. X. Tang, Proc. Natl. Acad. Sci. U.S.A. 103, 5764 (2006).

${ }^{18}$ M. Benoit, D. Gabriel, G. Gerisch, and H. E. Gaub, Nat. Cell Biol. 2, 313 (2000)

${ }^{19}$ X. H. Zhang, A. Chen, D. De Leon, H. Li, E. Noiri, V. T. Moy, and M. S. Goligorsky, Am. J. Physiol. Heart Circ. Physiol. 286, H359 (2003).

${ }^{20}$ V. Vogel and M. Sheetz, Nat. Rev. Mol. Cell Biol. 7, 265 (2006).

${ }^{21}$ C. Selhuber-Unkel, B. I. F. Futura 22, 197 (2007).

${ }^{22}$ T. Ludwig, R. Kirmse, K. Poole, and U. S. Schwarz, Pfluegers Arch. Eur. J. Physiol. 456, 29 (2008).

${ }^{23}$ C. M. Franz and P.-H. Puech, Cell Mol Bioeng 1, 289 (2008).

${ }^{24}$ A. Meister, M. Gabi, P. Behr, P. Studer, J. Vörös, P. Niedermann, J. Bitterli, J. Polesel-Maris, M. Liley, H. Heinzelmann, and T. Zambelli, Nano Lett. 9, 2501 (2009).

${ }^{25}$ F. Kühner, R. A. Lugmaier, S. Mihatsch, and H. E. Gaub, Rev. Sci. Instrum. 78, 075105 (2007).

${ }^{26}$ See supplementary material at http://dx.doi.org/10.1063/1.3462979 for the description of the custom FluidFM, the "before-after" images of the yeasts manipulation and the description of the "double-tube" tips microfabrication.

${ }^{27}$ S. Deladi, N. R. Tas, J. W. Berenschot, G. J. M. Krijnen, M. J. de Boer, J. H. de Boer, M. Peter, and M. C. Elwenspoek, Appl. Phys. Lett. 85, 5361 (2004).

${ }^{28}$ K. H. Kim, N. Moldovan, and H. D. Espinosa, Small 1, 632 (2005).

${ }^{29}$ T. S. Hug, T. Biss, N. F. de Rooij, and Q. Staufer Dig. Tech. Pap. Transducers, 2, 1191, (2005).

${ }^{30}$ A. Meister, J. Przybylska, P. Niedermann, C. Santschi, and H. Heinzelmann, NSTI-Nanotech 2008 (NSTI (Nano Science and Technology Institute, CRC, Taylor \& Francis Group, 2008), Vol. 3, pp. 273-276

${ }^{31}$ N. Kato, T. Kawashima, T. Shibata, T. Mineta, and E. Makino, Microelectronic Engineering 87, 1185 (2010).

${ }^{32}$ S. Deladi, J. W. Berenschot, N. R. Tas, G. J. M. Krijnen, J. H. de Boer, M. J. de Boer, and M. C. Elwenspoek, J. Micromech. Microeng. 15, 528 (2005).

${ }^{33}$ N. P. Huang, R. Michel, J. Vörös, M. Textor, R. Hofer, A. Rossi, D. L. Elbert, J. A. Hubbell, and N. D. Spencer, Langmuir 17, 489 (2001).

${ }^{34}$ The stepper motor was used to detach cells because of the limited maximal extension of the piezo. As consequence, a sinusoidal noise was superimposed to the $\mathrm{F}(\mathrm{z})$ curves.

${ }^{35}$ C. Selhuber-Unkel, T. Erdmann, M. Lopez-Garcia, H. Kessler, U. S. Schwarz, and J. P. Spatz, Biophys. J. 98, 543 (2010).

${ }^{36}$ G. Weder, N. Blondiaux, M. Giazzon, N. Matthey, M. Klein, R. Pugin, H. Heinzelmann, and M. Liley, Langmuir 26, 8180 (2010). 